\section{Loneliness Kills: Can Autonomous Systems and Robotics Assist in Providing Solutions?}

\section{Tauseef Gulrez ${ }^{1 *}$, Samia Neftimeziani ${ }^{1}$, Phil Mc evoy² and Anthony Hodgson ${ }^{3}$}

${ }^{1}$ Autonomous Systems and Advanced Robotics Centre, The School of Computing, Science and Engineering University of Salford, Manchester, United Kingdom ${ }^{2}$ Six Degrees Social Enterprise Greenwood Business Centre, Regent Road Salford, Manchester, United Kingdom

${ }^{3}$ Research and Development Salford Royal NHS Foundation Trust Salford, Manchester, United Kingdom

Loneliness Kills People who are lonely are at higher risk of death and people who are less lonely live longer. See, for example recent editorial comments "people with stronger social relationships had a $50 \%$ increased likelihood of survival than those with weaker social relationships" [1]. In the past decade, advances in robotics, artificial intelligence and embedded computing have enabled the application of robotics to many real-world environments [2-4].

What can this progress offer to address such a profound human need? Development of socially intelligent robotic systems to address social connectedness has become a topic of rapidly growing interest. Prototype solutions such as 'Giraff' are being trailed currently to deliver telepresence and social interaction to address issues such as loneliness, especially in the elderly [5].

Major European funding programmers for example the 'Active and Healthy ageing' in Horizon 2020 are beginning to stimulate more activity in the field of artificial intelligence and telepresence.

In the UK about $20 \%$ of the 64 million populations is aged over 65 years and feel moderately or severely socially isolated [6]. Loneliness is linked to risk of an earlier death [1,7] depression [8], dementia [9] and poor self-rated health [10]. Many factors have been identified that increase the risk of social isolation among the elderly, including physical or mental decline, relationship factors and low income. Many of these factors are difficult to prevent or to improve. Consequently, it is not easy to develop effective social isolation intervention programmes among the elderly population living under diverse conditions. A wide range of interventions have been tried to alleviate loneliness among the elderly, little is known, however, about the effectiveness of these interventions [6].

In this editorial, we introduce the concept of an autonomous assistive system (AAS), which aims to develop an unprecedented socially intelligent system, thereby helping to pave the way for the deployment of intelligent systems in settings such as homes, care homes and hospitals that require substantial human interaction.

Traditionally, in telepresence systems a human operator remotely interacts with people by guiding either a remotely located robot or through voice commands. The AAS project is developing a new socially intelligent system that will intervene and connect the resident with their social circles automatically. This innovative system will reduce the social isolation among people living with neurodegenerative disorders, e.g. Dementia, by detecting social isolation and recognizing loneliness by recognition of postural gestures and conversational analysis and then initiating appropriate responses.

The proposed AAS is a low-cost stand-alone autonomous interventional device to reduce social isolation and can easily be deployed at the usual place of residence of older people. The AAS has been developed to detect the isolation of the resident, based on the skeletal movements (bodies pose), facial expressions and speech data. For example if a resident maintains a fixed seated posture for long periods, with a posture correlated with a more depressed state the AAS will intervene and facilitate adoption of a socially engaging activity either directly or indirectly as appropriate for the situation. The AAS is built upon the commercially available Microsoft Kinect sensor and Autonomous Empowered Conversation. The Kinect has an RGB camera, infra-red (IR) depth sensor and microphone to enable skeletal, facial and speech tracking and recognition. The AAS has been augmented with a computer to process and store all the data collected by the Microsoft Kinect sensor.

In the future, we are planning to send the high density data over the cloud for the clinician to monitor in real- time and intervene remotely if appropriate; for example by initiating a home visit by a care worker. The Microsoft Kinect hardware was developed for the MS PlayStation and has powerful sensing technology and inbuilt software. It can be and has been developed into many healthcare innovations. Autonomous Empowered Conversation is an accessible new approach to interactive communication skills developed by Six Degrees Social Enterprise jointly with the University of Salford, Manchester. It is aimed at the day to day careers of people living with dementia, including both family careers and the wider dementia care support workforce. The Autonomous Empowered Conversations will intervene and act as a virtual career that will listen, learn and connect with people who are living with dementia by tuning into their experiences. The approach has been informed by the fields of applied linguistics, communication theory, perceptual control theory and psychodynamic psychotherapy. However, it can be distilled down into the two core concepts of empathic curiosity and dialogic engagement. Autonomous Empowered Conversations (Interventional System) has a speech emotion recognition system which identifies sadness, boredom, anger, neutral and happiness. This system utilizes the classification models by using acoustic features. We trained the models using different speech corpus. In addition, to resolve inter-speaker variations, we employed speaker adaptation techniques. This speech emotion recognition system produces labels, which might be possible subsets of features for higher levels of social states such as the quality of the conversation.

We hope that AAS will open a new chapter in defining a social intelligence arena in robotics for elderly care and will provide a robust socially intelligent behavior in a challenging real-world environment.

*Corresponding author: Tauseef Gulrez, Autonomous Systems and Advanced Robotics Centre, The School of Computing, Science and Engineering University of Salford, Manchester, United Kingdom, E-mail: T.Gulrez@salford.ac.uk

Received January 26, 2016; Accepted January 27, 2016; Published February 02,2016

Citation: Gulrez T, Neftimeziani S, Evoy PM, Hodgson A (2016) Loneliness Kills: Can Autonomous Systems and Robotics Assist in Providing Solutions? Int Swarm Intel Evol Comput 5: e113. doi: 10.4172/2090-4908.1000e113

Copyright: $\odot 2016$ Gulrez T, et al. This is an open-access article distributed under the terms of the Creative Commons Attribution License, which permits unrestricted use, distribution, and reproduction in any medium, provided the original author and source are credited. 
Citation: Gulrez T, Neftimeziani S, Evoy PM, Hodgson A (2016) Loneliness Kills: Can Autonomous Systems and Robotics Assist in Providing Solutions? Int J Swarm Intel Evol Comput 5: e113. doi: 10.4172/2090-4908.1000e113

Page 2 of 2

AAS will be able to recognize social signal inputs by combining various modalities such as speech, vision and depth sensors, with state-of-art algorithms. Having access to these multimodal social features will allow the system to act in a socially appropriate manner based on its embedded social cost functions.

\section{References}

1. Holt-Lunstad J, Smith TB, Layton JB (2010) Social relationships and mortality risk: a meta-analytic review. PLoS medicine 7: 859

2. Gulrez T, Tognetti A (2014) A sensorized garment controlled virtual robotic wheelchair. Journal of Intelligent and Robotic Systems 74: 847-868.

3. Pour PA, Gulrez T, Al Zoubi O, Gargiulo G, Calvo RA (2008) Brain- compute interface: Next generation thought controlled distributed video game development platform in Computational Intelligence and Games CIG '08. IEEE Symposium pp: 251-257.

4. Gulrez T (2014) Role of haptic interfaces in robot-assisted minimally invasive surgery. Int J Swarm Intel Evol Comput 4: e107.
5. Moyle C Jones W, Cooke M, Dwyer SO, Sung B, Drummond S (2014) Connecting the person with dementia and family: a feasibility study of a telepresence robot. BMC geriatrics 14: 7 .

6. Davidson S, Rossall P (2016) Evidence review: Loneliness in later life.

7. Penninx BW, Van Tilburg T, Kriegsman DM, Deeg DJ, Boeke AJP (1997) Effects of social support and personal coping resources on mortality in older age: the longitudinal aging study Amsterdam. American Journal of Epidemiology 146 510-519.

8. Musich S, Wang SS, Hawkins K, Yeh CS (2015) The impact of loneliness on quality of life and patient satisfaction among older, sicker adults. Gerontology and Geriatric Medicine.

9. Holwerda TJ, Deeg DJ, Beekman AT, Van Tilburg TG, Stek ML (2014) Feelings of loneliness, but not social isolation, predict dementia onset: results from the Amsterdam study of the elderly (amstel). Journal of Neurology, Neurosurgery and Psychiatry 85:135-142

10. Stickley A, Koyanagi B, Roberts E, Richardson P, Abbott S, et al. (2013) Loneliness: its correlates and association with health behaviours and outcomes in nine countries of the former Soviet Union. PloS one 8: 67978.
Citation: Gulrez T, Neftimeziani S, Evoy PM, Hodgson A (2016) Loneliness Kills: Can Autonomous Systems and Robotics Assist in Providing Solutions? Int J Swarm Intel Evol Comput 5: e113. doi: 10.4172/2090-4908.1000e113
OMICS International: Publication Benefits \& Features

\section{Unique features:}

Increased global visibility of articles through worldwide distribution and indexing

Showcasing recent research output in a timely and updated manner

Special issues on the current trends of scientific research

Special features:

700 Open Access Journal

50,000 editorial tean

Rapid review proces

Quality and quick editorial, review and publication processing

Indexing at PubMed (partial), Scopus, EBSCO, Index Copernicus and Google Scholar etc

Sharing Option: Social Networking Enabled

Authors, Reviewers and Editors rewarded with online Scientific Credits

Better discount for your subsequent articles

Submit your manuscript at: http://www.omicsonline.org/submission 